\title{
Treatment of renal cell carcinoma with a level III or level IV inferior vena cava thrombus using cardiopulmonary bypass and deep hypothermic circulatory arrest
}

Yong-Hui Chen ${ }^{1+}$, Xiao-Rong Wu ${ }^{1+}$, Zhen-Lei Hu², Wei-Jun Wang ${ }^{2}$, Chen Jiang ${ }^{1}$, Wen Kong ${ }^{1}$, Wei Chen ${ }^{1}$, Wei Xue ${ }^{1}$, Dong-Ming Liu ${ }^{1 *}$ and Yi-Ran Huang ${ }^{1 *}$

\begin{abstract}
Background: The aim of this study was to investigate the minimally invasive cardiopulmonary bypass (CPB) and deep hypothermic circulatory arrest (DHCA) approach in the management of renal cell carcinoma (RCC) with level III or IV inferior vena cava (IVC) thrombus and evaluate the survival outcomes.

Methods: We performed a retrospective analysis on 32 RCC patients with IVC thrombus that underwent nephrectomy and thrombectomy via the minimally invasive CPB/DHCA approach between January 2007 and December 2013. Perioperative variables (for example, operative time, CPB duration, and circulatory arrest duration), estimated blood loss, hospital stay, perioperative complications, and survival data were recorded and analyzed.

Results: Thirty-two patients (median age: 56 years) were treated surgically using the CPB and DHCA approach for RCC with a level III $(n=25)$ or level IV $(n=7)$ tumor thrombus. The median operation time was 360 min (interquartile range (IQR): 300 to $435 \mathrm{~min}$ ) with median CPB and DHCA durations of 149 min and 23 min, respectively. The median estimated blood loss was $2,500 \mathrm{ml}$. Four complications were observed but no deaths occurred perioperatively. The median follow-up was 25 months (range: 4 to 64 months). The mean overall survival (OS) was $28.2 \pm 4.6$ months while the disease-free survival (DFS) was $19.5 \pm 11.6$ months. In patients with M0 disease, ten patients developed metastases and were treated with sorafenib as an adjuvant therapy. The mean OS and DFS of this subgroup were $25.4 \pm 12.8$ months and $16.0 \pm 14.2$ months, respectively.

Conclusions: Radical nephrectomy and thrombectomy using CPB and DHCA to treat RCC is a relatively safe approach associated with low morbidity and mortality. This minimally invasive procedure may help minimize surgical trauma and improve perioperative outcomes.
\end{abstract}

Keywords: Cardiopulmonary bypass, Hypothermic arrest, Renal cell carcinoma, Thrombectomy, Tumor thrombus

\section{Background}

Renal cell carcinoma (RCC), one of the most common forms of genitourinary malignancy, accounts for $3 \%$ of all solid neoplasms [1]. Due to an increase in highresolution imaging, the incidence of $\mathrm{RCC}$ detection has increased. In approximately $20 \%$ to $30 \%$ of RCC cases,

\footnotetext{
* Correspondence: liudm541@126.com; yiranhuangrenji@163.com

${ }^{\dagger}$ Equal contributors

'Department of Urology, Ren Ji Hospital, School of Medicine, Shanghai Jiao Tong University, 1630 Dongfang Road, Pudong District, Shanghai 200127, China

Full list of author information is available at the end of the article
}

the patients either present with metastasis at the time of diagnosis or develop metastases after surgery [2]. Moreover, $4 \%$ to $10 \%$ of RCC patients also have a thrombus involving the inferior vena cava (IVC), and the tumor thrombus extends into the right atrium in $1 \%$ of these patients $[3,4]$.

The intravascular growth observed in RCC patients could signify a heightened or more aggressive biologic behavior of the tumor. However, some authors agree that the RCC-associated tumor thrombus does not translate to a specific prognostic significance if it can be treated

\section{Ciomed Central}

(c) 2015 Chen et al.; licensee BioMed Central. This is an Open Access article distributed under the terms of the Creative Commons Attribution License (http://creativecommons.org/licenses/by/4.0), which permits unrestricted use, distribution, and reproduction in any medium, provided the original work is properly credited. The Creative Commons Public Domain Dedication waiver (http://creativecommons.org/publicdomain/zero/1.0/) applies to the data made available in this article, unless otherwise stated. 
successfully with surgery [5]. To manage cases of RCC with IVC thrombus, several different surgical approaches such as liver transplantation [6,7] and thrombectomy using cardiopulmonary bypass $(\mathrm{CPB})$ and deep hypothermic circulatory arrest (DHCA) $[8,9]$ have been described. New developments in imaging, anesthesiology, and perioperative care have allowed for most thrombi to be removed successfully. There is, however, still considerable morbidity and mortality associated with treatment of this disease [10].

In the present study, we have retrospectively reviewed our management of RCC cases with level III and IV thrombi in a Chinese population using the minimally invasive CPB with DHCA approach. To the best of our knowledge, this study represents the largest analysis of surgical outcome in a Chinese population following this particular approach.

\section{Methods}

\section{Patients}

After Institutional Ethics Committee's approval, we retrospectively reviewed all level III and IV tumor thrombus RCC cases operated on in the Urology Department of Ren Ji Hospital at Shanghai Jiao Tong University from January 2007 to December 2013. Tumor thrombus staging was performed according to the guidelines established by Neves and Zincke [11]. In total, 32 patients were included in this study. All patients were preoperatively evaluated with ultrasound, computed tomography (CT), or magnetic resonance imaging (MRI). To evaluate metastases, additional imaging modalities such as chest radiography, brain and lung CTs, and bone scans or PET-CT were employed if necessary. Clinicopathological features, including gender, age, which kidney was involved, tumor size, histological type, and tumor grade were obtained and analyzed. The total operation time, duration of the DHCA, estimated blood loss, number of transfusions and complications were also evaluated.

One patient in the case series, a 51-year-old man, presented with right kidney RCC and an IVC thrombus extending to the level of the diaphragm. Magnetic resonance angiography accurately depicted the cranial extension of the neoplastic thrombus (Figure 1). Results of lung CT and bone scan were negative. The patient has given consent for the report to be published.

\section{Operative technique}

After the patient was placed in a supine position, a pararectal incision alongside the abdominal rectus incision (left: chevron incision) was made (Figure 2) and the abdomen was examined for evidence of tumor metastasis. The IVC and kidney were exposed, and the IVC was subsequently mobilized at the anterior surface only. An incision in the right groin was then made to expose the

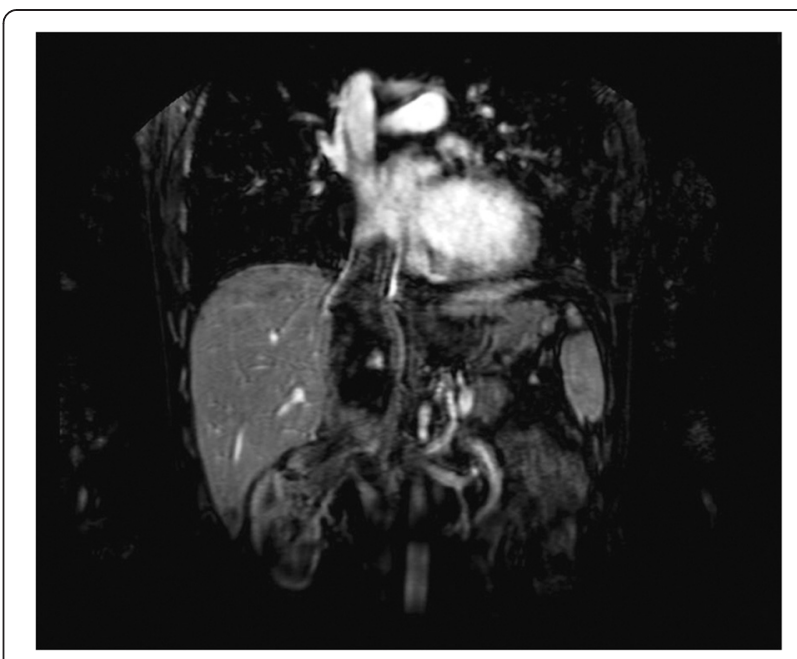

Figure $1 \mathrm{MRI}$ of a right-sided renal cell carcinoma with right atrial tumor thrombus.

right femoral artery, and a minithoracotomy was performed in the fourth intercostal space to open the pericardium. Pericardial stay sutures were then passed through and stabilized outside the chest. The patient was systemically heparinized. The femoral artery and the right atrium were cannulated. The patient was placed on $\mathrm{CPB}$ and cooled to $18^{\circ} \mathrm{C}$. DHCA was performed according to the protocol described by Welz et al. [12].

The IVC was mobilized as close to the diaphragm as possible, and the cephalad extension and the mobility of the tumor thrombus were confirmed using laparosonography. The venous collaterals, and the lumbar veins in particular, were ligated and sectioned, as hemorrhaging from these enlarged vessels is difficult to manage. After ligation of the lumbar veins, an anterior cavatomy was made to enable complete thrombus extraction. If necessary, an atriotomy was performed to gently milk the thrombus through the atriotomy into the IVC (Figure 3). After the thrombus was extracted from the IVC, laparoscopy or flexible cystoscopy was used to confirm complete tumor removal and whether the tumor was adherent to the IVC wall (Figure 4). If adherence was observed, a resection of the affected IVC segment was also performed.

Prior to closure, a small IVC cuff at the renal vein junction was also taken. The right atrium and IVC were closed with 5-0 Prolene running sutures. After caval reconstruction and closure of the right atrium, the $\mathrm{CPB}$ was reestablished and the patient was rewarmed to $36^{\circ} \mathrm{C}$. During this procedure, a radical nephrectomy was also performed (Figure 5).

\section{Results}

Between January 2007 to December 2013, 32 consecutive RCC cases (19 men, 13 women) with a level III $(n=25)$ or level IV $(n=7)$ tumor thrombus underwent thrombectomy 


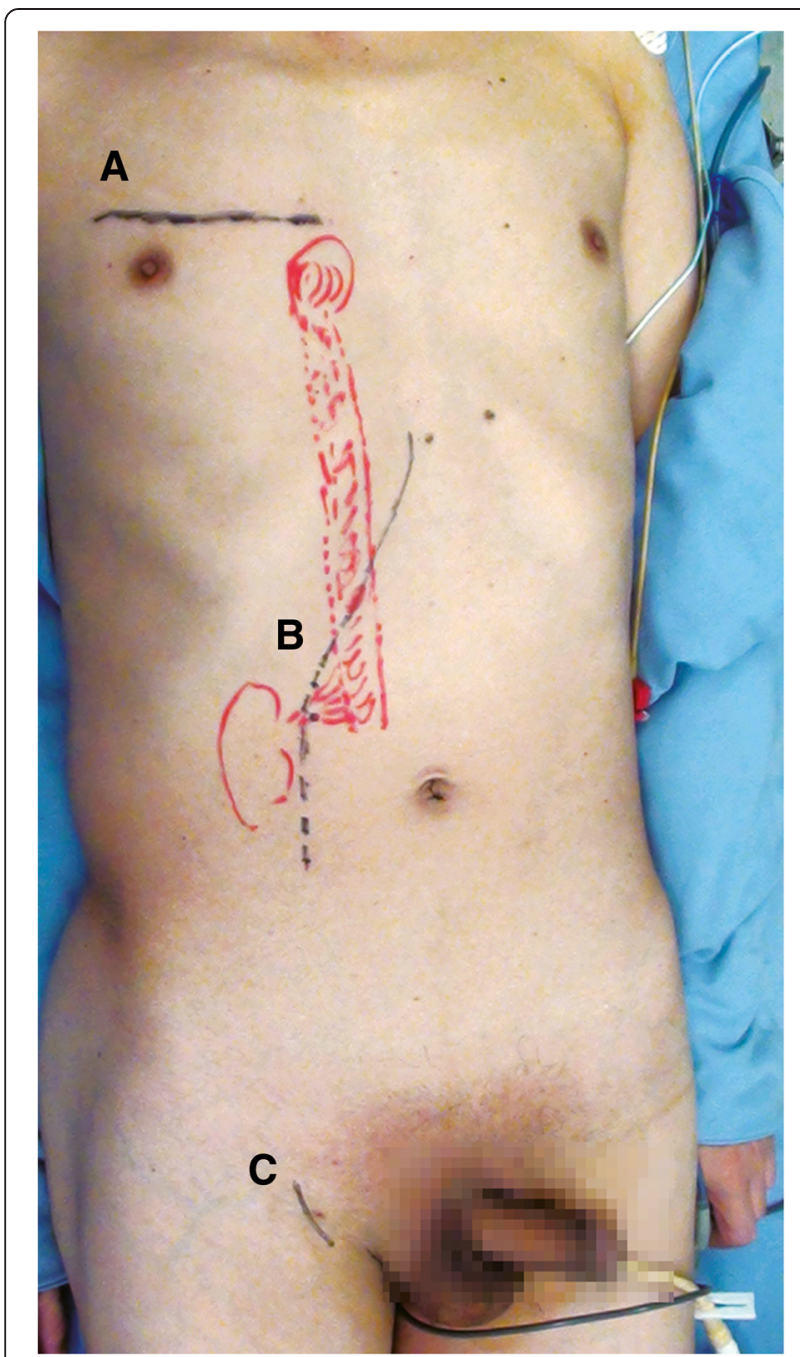

Figure $\mathbf{2}$ Incisions used for a right-sided tumor. An incision in the fourth intercostal space $(\mathbf{A})$ and the right groin $(\mathbf{C})$ were made to cannulate the superior vena cava and femoral artery. A subcostal incision alongside the abdominal rectus incision was made to expose the right renal and inferior vena cava (B).

via the minimally invasive $\mathrm{CPB}$ and DHCA approach (Table 1). At the time of surgery, the median age was 56 years (IQR: 48 to 62 years). In 12 (37.5\%) patients, the tumor was located on the left while 20 (62.5\%) patients had a right-sided tumor.

The median total operation time was $360 \mathrm{~min}$ with median CPB and DHCA durations of $149 \mathrm{~min}$ and 23 min, respectively. The median estimated blood loss was 2,500 $\mathrm{ml}$ (IQR: 1,650 to 4,500), and 31 patients required a blood transfusion, with a median estimated blood transfusion volume of 2,600 $\mathrm{ml}$ (IQR: 1,000 to $4,400)$. The decision to administer blood was based on the degree of blood loss and on preoperative hemoglobin levels.

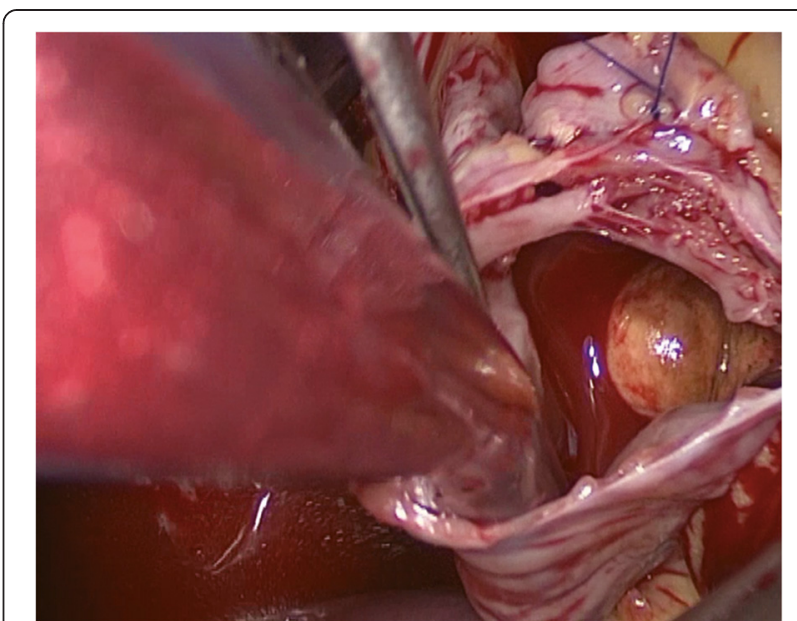

Figure 3 Atriotomy revealing a large atrial tumor thrombus.

Four complications were observed in this patient cohort: acute renal failure $(n=1)$, liver dysfunction $(n=1)$, cardiac failure $(n=1)$, and coagulopathy $(n=1)$. We did not observe any cases of tumor embolism and no mortalities occurred due to surgical complications. Patients remained in the hospital for a median of 7 days (range: 5 to 10 ), and 1.5 days in the intensive care unit (range: 1 to 3). Pathologic examination of the tumors revealed that $25(78.1 \%)$ patients had clear cell RCC, five (15.6\%) patients had papillary RCC, and two (6.3\%) patients had primitive neuroectodermal tumors (PENT). Three patients presented with tumor thrombi that invaded the IVC wall, such that part of the IVC luminal wall was resected. The longitudinal caval incision was closed with running sutures and none of the patients required IVC segment resection or IVC grafts. The median tumor size was $12 \mathrm{~cm}$ (range: 8 to 20).

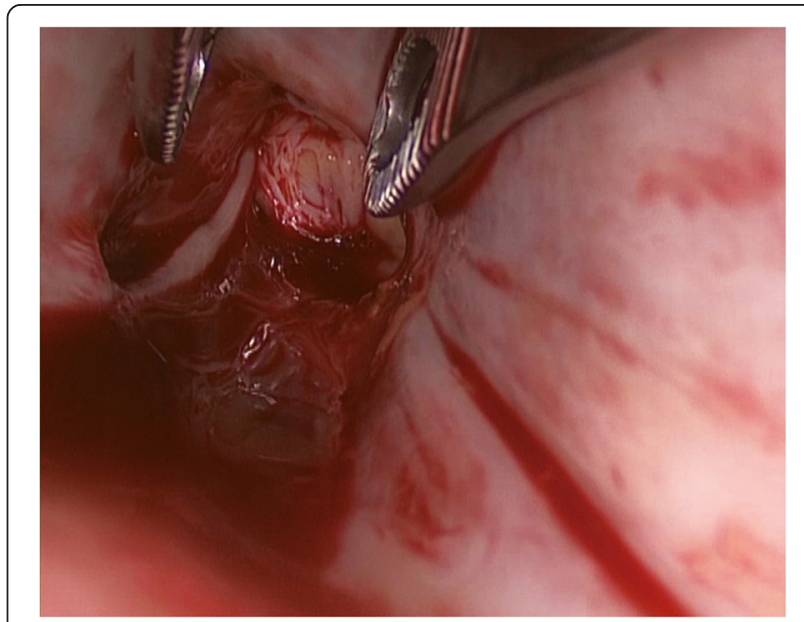

Figure $\mathbf{4}$ Laparoscopy was used to confirm the complete removal of the thrombus and determine whether the tumor was adherent to the IVC wall. 


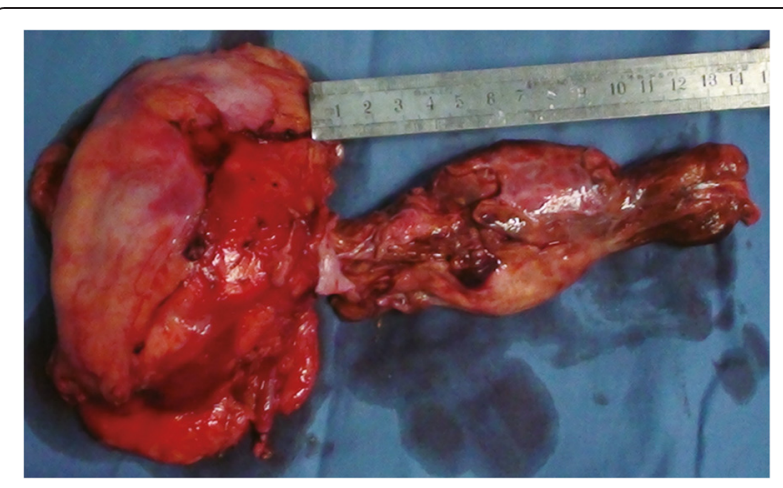

Figure $\mathbf{5}$ The specimen and tumor thrombus of a renal cell carcinoma patient with right atrial tumor thrombus invasion.

The median follow-up for the patients was 25 months (range: 4 to 64). The mean overall survival (OS) period of the cohort was $28.2 \pm 4.6$ months, while the diseasefree survival (DFS) period was $19.5 \pm 11.6$ months. Two patients $(6.25 \%)$ in our population had M1 disease, which is lower than what has been reported in other previously published cohorts, and both of these patients died. The mean OS of the two M1 patients was 4.5 months. In the patients with M0 disease, ten (33.3\%) developed metastases. All patients with metastases were

Table 1 Baseline characteristics of the patients

\begin{tabular}{|c|c|}
\hline Parameters & Values \\
\hline Number of patients, $n$ & 32 \\
\hline Median age $(I Q R)$, years & 56 (48 to 62$)$ \\
\hline Male/female, $n$ & $19 / 13$ \\
\hline Left/right kidney, $n$ & $12 / 20$ \\
\hline Thrombus level III/IV, $n$ & $25 / 7$ \\
\hline Median tumor size (range), centimeters & 12 (8 to 20$)$ \\
\hline \multicolumn{2}{|l|}{ Median (IQR) duration of } \\
\hline Surgery, minutes & 360 (300 to 435) \\
\hline $\mathrm{CPB}$, minutes & 149 (138 to 177$)$ \\
\hline DHCA, minutes & 23 (17 to 32$)$ \\
\hline Patients requiring IVC grafts, $n$ & 0 \\
\hline Median hospital stay (range), days & $7(5$ to 10$)$ \\
\hline Estimated blood loss (IQR), milliliters & $2,500(1,650$ to 4,000$)$ \\
\hline \multicolumn{2}{|l|}{ Tumor stage } \\
\hline pT3b & 24 \\
\hline pT3c & 5 \\
\hline pT4 & 3 \\
\hline \multicolumn{2}{|l|}{ Tumor pathology } \\
\hline Clear cell & 25 \\
\hline Papillary & 5 \\
\hline PENT & 2 \\
\hline
\end{tabular}

CPB, cardiopulmonary bypass; DHCA, deep hypothermic circulatory arrest; IQR, interquartile range; PENT, primitive neuroectodermal tumor. administered sorafenib as an adjuvant therapy. The mean OS and DFS of this subgroup were $25.4 \pm 12.8$ months and $16.0 \pm 14.2$ months, respectively. The mean OS of patients without metastases was $33.0 \pm 18.7$ months.

\section{Discussion}

Medical therapies have proven ineffective in the treatment of RCC with IVC thrombosis, and radical nephrectomy together with thrombectomy is the only effective therapeutic alternative for these patients $[9,13]$. Surgical management of RCC in which there is extension into the renal vein, IVC, or even the right atrium, however, poses a significant technical challenge [10]. With the technological advances in imaging, anesthesiology, and cardiosurgery, surgical treatment of RCC with an IVC thrombus is now feasible, and the thrombus can almost always be removed successfully.

Although the benefit of $\mathrm{CPB}$ and DHCA in RCC patients with level IV thrombus is obvious, controversy still persists in the treatment of RCC patients with a level III thrombus. Some experts suggest the use of nonextracorporeal circulation and hypothermic circulatory arrest to facilitate the removal of the tumor thrombus [6,7]. High level thrombi can also be managed with venovenous bypass via a caval-atrial shunt [14]. This technique avoids $\mathrm{CPB}$ and circulatory arrest, but it may result in hepatic venous bleeding even when performed by experienced surgeons. At our institution, we prefer an extracorporeal circulation approach, as the use of CPB and DHCA decrease the risk for unexpected and life-threatening intraoperative hemorrhage and incomplete tumor extirpation, which are the primary goals of the surgery. With help from the Department of Cardiac Surgery, CPB and DHCA have been used to treat RCC with level III or IV IVC thrombi in our hospital since 1997.

We have made some technical modifications to this surgical approach in comparison to the published procedure [12]. First, after a pararectal (for right RCC) or chevron incision (for left RCC) was made and the metastases were assessed, only the anterior part of the IVC was mobilized to reduce kidney mobilization to prevent tumor embolism. Second, none of the patients in this cohort underwent preoperative renal artery embolization, as it is associated with increased complications and perioperative mortality [13]. Third, a minithoracotomy was performed in the fourth intercostal space in lieu of a median sternotomy to open the pericardium. A minithoracotomy allows for a rapid postoperative recovery and reduces the use of analgesics and the length of hospital stay [15]. As previously reported [8], because the minithoracotomy has only a small incision, there are esthetic advantages. This technique is faster and has fewer requirements for mechanical ventilation and transfusion than traditional median sternotomy $[8,9]$. 
In this patient cohort, after $\mathrm{CPB}$ was established and during the procedure of cooling the body to a core temperature of $18^{\circ} \mathrm{C}$, the IVC was mobilized. Once DHCA was achieved, the thrombectomy was performed. After vascular reconstruction was complete, the patient was rewarmed. During the rewarming period, a radical nephrectomy and lymphadenectomy were performed. Performing the nephrectomy and lymphadenectomy during the rewarming period reduces the waiting time during the cardiosurgical portion of the procedure and shortens the total operation time.

IVC thrombus is often associated with tumor metastasis. Most of the previously published cohorts report that $30 \%$ of patients have M1 disease [3], and IVC thrombectomy is advocated as it may relieve the symptoms and potentially allow for a better quality of life $[3,10]$. Postoperative targeted adjuvant therapy with either sorafenib or sunitinib in patients with clear cell RCC, who are at a high risk for disease recurrence, and it is effective in reducing the recurrence rate [16]. This promising result warrants further analysis in randomized clinical trials with larger patient populations.

Our study has several limitations. It was performed retrospectively in a single institution, and there was some bias in patient selection. The study population was small and the number of patients with M1 disease was lower than other published series $[3,8]$. Future studies should be conducted to compare this technique with other surgical techniques for the management of RCC with a level III or IV tumor thrombus.

\section{Conclusions}

Patients with level III or IV IVC thrombus RCC can be treated safely and effectively via radical nephrectomy and thrombectomy using CPB combined with DHCA. This approach is associated with low rates of morbidity and mortality. Introduction of a right anterior minithoracotomy may minimize surgical trauma, accelerate postoperative recovery, and improve perioperative outcomes.

\footnotetext{
Abbreviations

CPB: cardiopulmonary bypass; DHCA: deep hypothermic circulatory arrest; IVC: inferior vena cava; PENT: primitive neuroectodermal tumors; RCC: renal cell carcinoma.
}

\section{Competing interests}

The authors declare that they have no competing interests.

\section{Authors' contributions}

YHC and XRW performed the investigation and wrote the manuscript. ZLH, WJW, CJ, WK, WC, and WX performed the data collection. DML and YRH designed the study and helped to draft the manuscript. All authors read and approved the final manuscript.

\section{Acknowledgements}

This work was supported in part by Award Number $20135 Y 027$ from the Shanghai Municipal Commission of Health and Family Planning and Award Number 2012206 from the Bureau of Health of Shanghai.

\section{Author details}

'Department of Urology, Ren Ji Hospital, School of Medicine, Shanghai Jiao Tong University, 1630 Dongfang Road, Pudong District, Shanghai 200127, China. ${ }^{2}$ Department of Cardiovascular Surgery, Ren Ji Hospital, School of Medicine, Shanghai Jiao Tong University, 1630 Dongfang Road, Pudong District, Shanghai 200127, China.

Received: 1 December 2014 Accepted: 14 April 2015

Published online: 22 April 2015

\section{References}

1. Jemal A, Siegel R, Xu J, Ward E. Cancer statistics, 2010. CA Cancer J Clin. 2010;60(5):277-300.

2. Janzen NK, Kim HL, Figlin RA, Belldegrun AS. Surveillance after radical or partial nephrectomy for localized renal cell carcinoma and management of recurrent disease. Urol Clin North Am. 2003;30(4):843-52.

3. Ciancio G, Manoharan M, Katkoori D, De Los SR, Soloway MS. Long-term survival in patients undergoing radical nephrectomy and inferior vena cava thrombectomy: single-center experience. Eur Urol. 2010;57(4):667-72.

4. Marshall FF, Dietrick DD, Baumgartner WA, Reitz BA. Surgical management of renal cell carcinoma with intracaval neoplastic extension above the hepatic veins. J Urol. 1988;139(6):1166-72.

5. Bachmann A, Seitz M, Graser A, Reiser MF, Schafers HJ, Lohe F, et al. Tumour nephrectomy with vena cava thrombus. BJU Int. 2005;95(9):1373-84.

6. Ciancio G, Livingstone AS, Soloway M. Surgical management of renal cell carcinoma with tumor thrombus in the renal and inferior vena cava: the University of Miami experience in using liver transplantation techniques. Eur Urol. 2007:51(4):988-94. discussion 994-985.

7. Ciancio G, Soloway MS. Renal cell carcinoma with tumor thrombus extending above diaphragm: avoiding cardiopulmonary bypass. Urology. 2005;66(2):266-70.

8. Wotkowicz C, Libertino JA, Sorcini A, Mourtzinos A. Management of renal cell carcinoma with vena cava and atrial thrombus: minimal access vs median sternotomy with circulatory arrest. BJU Int. 2006;98(2):289-97.

9. Bertini R, Roscigno M, Lapenna E, Pasta A, Petralia G, Strada E, et al. Radical nephrocapsulectomy and caval thrombectomy with extracorporeal circulation and deep hypothermic circulatory arrest in right anterior minithoracotomy: a minimally invasive approach. Urology. 2008;71(5):957-61.

10. Kirkali Z, Van Poppel H. A critical analysis of surgery for kidney cancer with vena cava invasion. Eur Urol. 2007;52(3):658-62.

11. Neves RJ, Zincke H. Surgical treatment of renal cancer with vena cava extension. Br J Urol. 1987;59(5):390-5.

12. Welz A, Schmeller N, Schmitz C, Reichart B, Hofstetter A. Resection of hypernephromas with vena caval or right atrial tumor extension using extracorporeal circulation and deep hypothermic circulatory arrest: a multidisciplinary approach. Eur J Cardiothorac Surg. 1997;12(1):127-32.

13. Subramanian VS, Stephenson AJ, Goldfarb DA, Fergany AF, Novick AC, Krishnamurthi V. Utility of preoperative renal artery embolization for management of renal tumors with inferior vena caval thrombi. Urology. 2009;74(1):154-9.

14. Foster RS, Mahomed Y, Bihrle R, Strup S. Use of cavalatrial shunt for resection of caval tumor thrombus in renal cell carcinoma. J Urol. 1988;140(6):1370-1.

15. Grossi EA, Galloway AC, LaPietra A, Ribakove GH, Ursomanno P, Delianides J, et al. Minimally invasive mitral valve surgery: a 6-year experience with 714 patients. Ann Thorac Surg. 2002;74(3):660-3. discussion 663-664.

16. Zhao J, Zhu Y, Zhang C, Wang X, He H, Wang H, et al. Sorafenib or sunitinib as postoperative adjuvant therapy for Chinese patients with locally advanced clear cell renal cell carcinoma at high risk for disease recurrence. Urol Oncol. 2013;31(8):1800-5. 